\title{
Global Suicide Rates and Climatic Temperature
}

\author{
Yusuke Arima ${ }^{1 *}$ \\ yusukearima77@gmail.com \\ Hideki Kikumoto ${ }^{2}$ \\ kkmt@iis.u-tokyo.ac.jp
}

\begin{abstract}
Global suicide rates vary by country ${ }^{1}$, yet the cause of this variability has not yet been explained satisfactorily ${ }^{2,3}$. In this study, we analyzed averaged suicide rates ${ }^{4}$ and annual mean temperature in the early $21^{\text {st }}$ century for 183 countries worldwide, and our results suggest that suicide rates vary with climatic temperature. The lowest suicide rates were found for countries with annual mean temperatures of approximately $20{ }^{\circ} \mathrm{C}$. The correlation suicide rate and temperature is much stronger at lower temperatures than at higher temperatures. In the countries with higher temperature, high suicide rates appear with its temperature over about $25^{\circ} \mathrm{C}$. We also investigated the variation in suicide rates with climate based on the Köppen-Geiger climate classification ${ }^{5}$, and found suicide rates to be low in countries in dry zones regardless of annual mean temperature. Moreover, there were distinct trends in the suicide rates in island countries. Considering these complicating factors, a clear relationship between suicide rates and temperature is evident, for both hot and cold climate zones, in our dataset. Finally, low suicide rates are typically found in countries with annual mean temperatures within the established human thermal comfort range. This suggests that climatic temperature may affect suicide rates globally by effecting either hot or cold thermal stress on the human body.
\end{abstract}

\section{KEYWORDS}

Suicide rate, Climatic temperature, Human thermal comfort, Köppen-Geiger climate classification

\footnotetext{
Affiliation:

${ }^{1}$ Department of Architecture, Polytechnic University of Japan, Tokyo, Japan.

${ }^{2}$ Institute of Industrial Science, the University of Tokyo, Tokyo, Japan.

${ }^{*}$ Corresponding author
} 


\section{MAIN TEXT}

Suicide rates vary globally but tend to remain temporally stable in each country ${ }^{1}$. Although suicide can be considered an individual act, suicide rates have been analyzed in relation to both socioeconomic $^{2}$ and meteorological ${ }^{3}$ factors. However, the dominant factors controlling variations in suicide rates have not yet been constrained. Year-to-year variations in suicide rates within particular regions or countries can be explained, to some extent, by socio-economic factors such as economic crises $^{6}$. However, as Rehkopf \& Buka $^{2}$ revealed in a systematic review, the relationship between suicide rates and socio-economic factors is less pronounced in cross-sectional studies than in timeseries (longitudinal) studies, and uncertainty in this relationship increases with increasing scale of the region examined. For example, almost all community-level studies undertaken to date have reported inverse relationships with lower rates of suicide in higher socio-economic areas, yet only half of similar country-level studies have demonstrated such a relationship ${ }^{2}$. In cross-sectional analysis at the global level, the high suicide rates in wealthy countries (such as the Nordic states) makes it difficult to explain differences in global suicide rates based on socio-economic factors alone.

Suicide rates have previously been investigated with reference to meteorological conditions ${ }^{3}$. The seasonal variation in suicide rates has, in particular, been seen as evidence supporting the idea of a relationship between suicide and meteorological factors ${ }^{7,8}$. Recent studies have reported an increase in the number of suicides occurring on days with high temperatures ${ }^{9}$, which is of interest to researchers as it suggests that climate change could increase the risk of suicide. Conversely, in a cross-sectional analysis of Europe, Fountoulakis et al. ${ }^{10}$ reported that countries with lower annual mean temperatures tended to have higher suicide rates and that low temperatures predict suicide rates more accurately than GDP per capita. These seemingly conflicting results may indicate that the effects of temperature on suicide rates depend on the temporal scale of change being considered. More specifically, weather temperature (i.e., daily or seasonal fluctuations in temperature) and climatic temperatures (such as that represented by annual mean temperature) could have different effects on suicide rates.

To clarify the relationship between suicide rates and climatic temperature, we analyzed differences between suicide rates and climate in 183 countries worldwide, focusing primarily on annual mean temperature. Annual mean temperatures were obtained for each country by taking the mean of the period 2000-2009 from Meteonorm ${ }^{11}$, a global meteorological database. Temperature data from the most populous city in each country, or from another appropriate city were used, the latter when no observations were available for the former. All of the stations selected can be identified using the World Meteorological Organization (WMO) station index number (Extended Data Table 1). We used suicide rate data (per 100,000 people) at the country level, as published by the World Health Organization $(\mathrm{WHO})^{4}$ for the years $2000,2005,2010,2015$, and 2016, and the mean values calculated 
from these data. The climate and suicide rate data do not cover exactly the same periods; however, we decided to include data from as many years as possible to best capture the average suicide rate. In addition, we used country-level gross domestic product (GDP) per capita for the years 2000-2016, as published by the World Bank ${ }^{12}$, as an indicator of the economic characteristics of each country. Additionally, we classified the most populous cities in each country by climate zones following the Köppen-Geiger climate classification for the 50-year period 1951-20005.

Figure 1 illustrates the relationship between suicide rates and annual mean temperatures in countries worldwide. Suicide rates reach a minimum at $\sim 20{ }^{\circ} \mathrm{C}$ and broadly increase as temperatures both increase and decrease from there. A linear correlation between suicide rate and temperature is evident for low temperatures. High suicide rates occur in a number of countries with annual mean temperatures exceeding $25^{\circ} \mathrm{C}$, although any correlation between these factors is not very clear. Similar trends were observed in both males and females, although the correlation is stronger for males (Extended Data Fig. 1). Additionally, the overall distribution of global suicide rates cannot be explained by GDP per capita alone (Fig. 1; see also Extended Data Fig. 2).

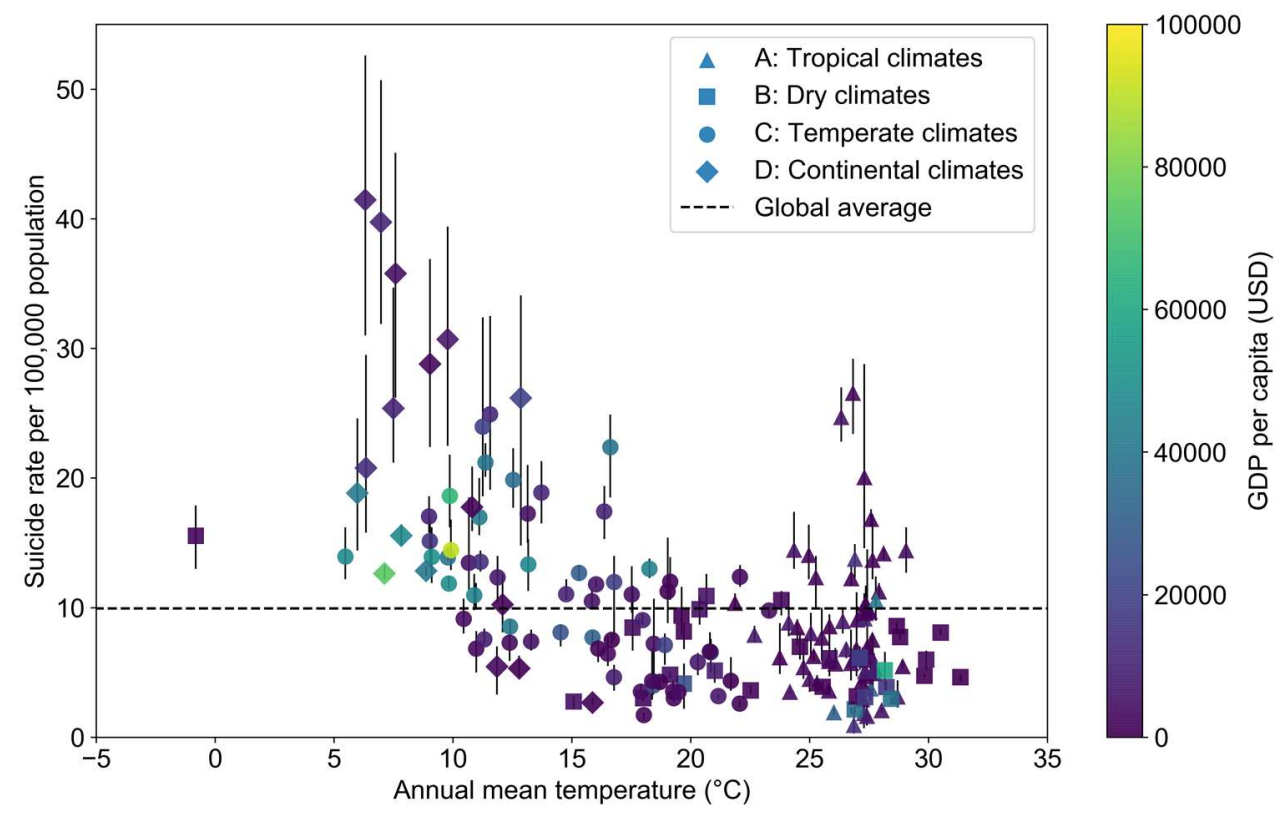

Fig. 1| Correlation of annual mean temperature and suicide rate for both sexes globally. Countries are categorized based on the Köppen-Geiger classification and denoted by different markers colored according to the average GDP per capita for the years considered. Error bars indicate minimum-maximum range in annual suicide rate for each country. The broken line shows global average suicide rate ( 9.94 per 100,000 people). 
To investigate the relationship between temperature and suicide rate in more detail, we also classified our dataset according to the Köppen-Geiger climate classification ${ }^{5}$. We found a weak correlation between temperature and suicide rate, particularly in dry zone countries (Fig. 1). This could be due to the influence of climatic factors apart from temperature, such as humidity. For example, the correlation between temperature and absolute humidity (water vapor pressure) is very weak in dry zone countries, which is contrary to that for other climatic zones (Extended Data Fig. 3). Moreover, we found that suicide rates in island countries exhibit a different pattern to those in other countries with similar annual mean temperatures, which suggests that it is necessary to consider geographical features unique to those countries. When dry zone countries and island countries are excluded, the correlation between suicide rate and temperature is much stronger for both hot and cold regions (Extended Data Fig. 4).

The range in annual mean temperature at which suicide rates are low coincides with the temperature range that has been established as suitable for human thermal comfort; the $\mathrm{WHO}^{13}$ states that $18-24{ }^{\circ} \mathrm{C}$ is a comfortable and healthy temperature range for the human body. Ambient temperature and climatic annual mean temperature cannot be considered equivalent owing to the effects of daily or seasonal temperature fluctuations. However, annual mean temperatures can be considered representative of the length of time for which people resident in a given country may be exposed to a comfortable range of temperatures. Therefore, the correspondence between low suicide rates and the temperature range for human thermal comfort suggests that climatic temperature could affect suicide rates by inducing thermal stress in both hot and cold climates.

Of course, climatic temperature alone cannot explain all variations in global suicide rates; investigating other factors create these differences is also important. However, an improved understanding of the relationship between suicide rates and climatic temperature offered by the present study could help with analyzing the effects of other factors. For example, suicide rates in Nordic countries are higher than global average; previously, this has been attributed social issues ${ }^{1}$. However, our results demonstrate that these suicide rates are relatively lower than those in other countries with similarly severe cold climates (Fig. 1). The latter could be explained in terms of economic wealth (based on GDP per capita), as wealthier populations can more easily procure necessities to protect them against cold. In order to make better use of these findings, the relationship between suicide rates, socio-economic factors, and climatic temperature should be analyzed in large-scale cross-sectional studies covering a larger region.

There are still several suicide phenomena related to meteorological factors that remain to be understood. For example, suicide seasonality exhibits a pronounced peak during spring or early 
summer $^{8}$, and recent previous studies have demonstrated the possibility of a positive relationship between high temperature and high suicide rates ${ }^{9}$. It seems that these phenomena cannot be interpreted as effects of thermal comfort because similar phenomena have also been observed in countries that are generally $\operatorname{cold}^{7}$. If the relationship between suicide and temperature were just a matter of thermal comfort, the number of suicides would decrease on warm days or seasons in cold countries. Therefore, we suggest that the effects of temperature on suicide rates may vary depending on the temporal scale of the change being considered and that different causal mechanisms may be responsible at different scales. Moreover, the lack of any clear relationship between suicide rates and temperature in dry zones suggests that other meteorological factors may be important; for example, humidity is known to be important for both thermal comfort and human health in general ${ }^{14}$. We posit that the relationship between suicide rate and temperature presented here is likely part of a broader, more complex mechanism that includes the interaction of temperature with other meteorological factors. Furthermore, we acknowledge that suicide is not affected only by climate, but is a complex phenomenon involving geographical, social and other factors, and the contributions of these merit further investigation. Nevertheless, we believe that we have, for the first time, demonstrated the link between climatic temperature and suicide rates on a global scale. Our findings help to better explain the various factors affecting global suicide rates. 


\section{REFERENCES}

1. Värnik, P. Suicide in the world. International Journal of Environmental Research and Public Health. 9(3), 760-771 (2012).

2. Rehkopf, D.H. \& Buka, S.L. The association between suicide and the socio-economic characteristics of geographical areas: A systematic review. Psychological Medicine. 36, 145157 (2006).

3. Dixon, P.G. \& Kalkstein, A.J. Climate-suicide relationships: A research problem in need of geographic methods and cross-disciplinary perspectives. Geography Compass. 3, 1-14 (2009).

4. World Health Organization. The Global Health Observatory-Crude suicide rates (per 100000 population).

https://www.who.int/data/gho/data/indicators/indicator-details/GHO/crude-suicide-rates-(per100-000-population) (Accessed: 2020-05-25)

5. Kottek, M. et al. World map of the Köppen-Geiger climate classification updated. Meteorologische Zeitschrift. 15(3), 259-263 (2006).

6. Chang, S.-S. et al. Was the economic crisis 1997-1998 responsible for rising suicide rates in East/Southeast Asia? A time-trend analysis for Japan, Hong Kong, South Korea, Taiwan, Singapore and Thailand. Social Science \& Medicine. 68(7), 1322-1331 (2009).

7. Kim, Y. et al. Suicide and ambient temperature: A multi-country multi-city study. Environmental Health Perspectives. 127(11), 117007-1-1700-10 (2019).

8. Christodoulou, C. et al. Suicide and seasonality. Acta Psychiatrica Scandinavica. 125 (2), $127-$ 146 (2011).

9. Burke, M. et al. Higher temperatures increase suicide rates in the United States and Mexico. Nature Climate Change. 8, 723-729 (2018).

10. Fountoulakis, K.N. et al. Relationship of suicide rates with climate and economic variables in Europe during 2000-2012. Annals of General Psychiatry. 15(19), (2016).

11. Meteonorm 7.3. http://www.meteonorm.com/ (Accessed: 2020-05-25)

12. The World Bank. World Bank Open Data-GDP per capita (current US\$). https://data.worldbank.org/indicator/NY.GDP.PCAP.CD (Accessed: 2020-05-25)

13. World Health Organization. Housing impacts health: New WHO Guidelines on Housing and health guidelines. (2018).

14. Davis, R.E., McGregor, G.R. \& Enfield, K.B. Humidity: A review and primer on atmospheric moisture and human health. Environmental Research. 144, 106-116 (2016). 


\section{METHODS}

All data sets used for analysis in this paper are publicly available. We collected data from the WHO Global Health Observatory ${ }^{4}$ and the World Bank Open Data ${ }^{12}$, and Meteonorm $7.3^{11}$. Detailed information for each data set is provided in the paper (Extended Data Table 1).

\section{EXTENDED DATA}

All country data used in our study are presented below in Extended Data Table 1. For suicide rates in each year, see the data published by $\mathrm{WHO}^{4}$. Some supplementary figures (Extended Data Fig. 1-4) are also provided to help demonstrate the scientific value and authenticity of our results to reviewers.

Extended Data Table 1|All data used in this study

\begin{tabular}{|c|c|c|c|c|c|c|c|c|c|}
\hline \multirow[t]{2}{*}{ Country } & \multicolumn{3}{|c|}{$\begin{array}{l}\text { Averaged suicide rates } \\
\text { for } 2000,2005,2010, \\
2015 \text { and } 2016 \\
\text { (per } 100,000 \text { people) }\end{array}$} & \multirow{2}{*}{\begin{tabular}{|c|} 
Annual \\
mean \\
temperatu \\
re for \\
$2000-$ \\
$2009\left[{ }^{\circ} \mathrm{C}\right]$ \\
\end{tabular}} & \multicolumn{2}{|c|}{$\begin{array}{l}\text { The city at which the weather data } \\
\text { we used }(*)\end{array}$} & \multirow[t]{2}{*}{\begin{tabular}{|c|} 
GDP per \\
capita [USD]
\end{tabular}} & \multirow[t]{2}{*}{\begin{tabular}{|c|} 
Köppen- \\
Geiger \\
climate \\
classification
\end{tabular}} & \multirow[t]{2}{*}{$\begin{array}{c}\text { Island } \\
\text { country }\end{array}$} \\
\hline & $\begin{array}{l}\text { Both } \\
\text { sexes }\end{array}$ & Male & Female & & City & $\begin{array}{l}\text { WMO station } \\
\text { index number }\end{array}$ & & & \\
\hline Afghanistan & 5.32 & 8.96 & 1.38 & 12.8 & Kabul & 409480 & 427 & Dsb & \\
\hline Albania & 6.46 & 8.02 & 4.88 & 16.5 & Tirana & 136150 & 3272 & $\mathrm{Csa}$ & \\
\hline Algeria & 3.52 & 5.06 & 1.98 & 17.9 & Dar El Beïda & 603900 & 3764 & $\mathrm{Csa}$ & \\
\hline Angola & 6.1 & 9.22 & 3.12 & 25.8 & Luanda & 661600 & 2980 & BSh & \\
\hline Antigua and Barbuda & 0.96 & 1.42 & 0.5 & 26.9 & Coolidge* & 788620 & 13010 & Af & Yes \\
\hline Argentina & 9.04 & 14.78 & 3.56 & 18.0 & Buenos Aires & 875820 & 8763 & $\mathrm{Cfa}$ & \\
\hline Armenia & 5.46 & 9.06 & 2.24 & 11.8 & Yerevan & 377890 & 2566 & Dfa & \\
\hline Australia & 13 & 19.5 & 6.52 & 18.3 & Sydney & 947680 & 43438 & $\mathrm{Cfa}$ & \\
\hline Austria & 16.98 & 26.08 & 8.36 & 11.1 & Vienna & 110350 & 41692 & $\mathrm{Cfb}$ & \\
\hline Azerbaijan & 2.76 & 4.44 & 1.12 & 15.1 & Baku & 378640 & 4009 & BSk & \\
\hline Bahamas & 1.94 & 3.32 & 0.6 & 26.0 & Nassau & 780730 & 29351 & Aw & Yes \\
\hline Bahrain & 6.14 & 8.58 & 2.42 & 27.1 & Muharraq* & 411500 & 19542 & $\mathrm{BWh}$ & Yes \\
\hline Bangladesh & 6.28 & 4.78 & 7.76 & 25.2 & Dhaka & 149170 & 726 & Aw & \\
\hline Barbados & 1.88 & 2.52 & 1.26 & 27.3 & Bridgetown & 809960 & 14911 & $\mathrm{Am}$ & Yes \\
\hline Belarus & 35.78 & 63.72 & 11.36 & 7.6 & Minsk & 268500 & 4620 & $\mathrm{Dfb}$ & \\
\hline Belgium & 21.2 & 29.32 & 13.38 & 11.4 & Antwerp & 64500 & 38995 & $\mathrm{Cfb}$ & \\
\hline Belize & 4.96 & 8.16 & 1.82 & 27.4 & Belize City & 785830 & 4178 & $\mathrm{Am}$ & \\
\hline Benin & 9.72 & 13.58 & 5.98 & 27.5 & Cotonou & 653440 & 687 & $\mathrm{Aw}$ & \\
\hline Bhutan & 11.74 & 14.32 & 8.88 & None & None & None & 1842 & $\mathrm{Cwb}$ & \\
\hline Bolivia & 14.08 & 17.72 & 10.38 & 25.0 & $\begin{array}{c}\text { Sant Cruz de la } \\
\text { Sierra }\end{array}$ & 852450 & 1814 & Aw & \\
\hline Bosnia and Herzegovina & 9.14 & 14.72 & 3.74 & 10.5 & Sarajevo & 146540 & 3817 & $\mathrm{Cfb}$ & \\
\hline Botswana & 10.9 & 17.24 & 4.7 & 20.7 & Gaborne & 682400 & 5777 & $\mathrm{BSh}$ & \\
\hline Brazil & 5.8 & 8.94 & 2.72 & 20.3 & São Paulo & 837800 & 7691 & $\mathrm{Cfa}$ & \\
\hline Brunei Darussalam & 3.78 & 5.36 & 2.08 & 27.6 & $\begin{array}{c}\text { Bandar Seri } \\
\text { Begawan }\end{array}$ & 963150 & 30723 & Af & Yes \\
\hline Bulgaria & 13.46 & 20.64 & 6.62 & 10.7 & Sofia & 156140 & 5411 & $\mathrm{Cfb}$ & \\
\hline Burkina Faso & 7.74 & 10.6 & 4.98 & 28.8 & Quagadougou & 655030 & 490 & $\mathrm{BSh}$ & \\
\hline Burundi & 8.5 & 12.06 & 5.04 & 24.5 & Bujumbura & 633900 & 200 & Aw & \\
\hline Cabo Verde & 10.58 & 15.16 & 6.12 & 23.8 & Sal Island** & 85940 & 2742 & $\mathrm{BWh}$ & Yes \\
\hline Cambodia & 5.5 & 7.88 & 3.24 & 28.9 & Phnom Penh & 489910 & 708 & $\mathrm{Am}$ & \\
\hline Cameroon & 12.28 & 17.02 & 7.54 & 26.7 & Douala & 649100 & 1152 & $\mathrm{Am}$ & \\
\hline Canada & 12.84 & 18.88 & 6.9 & 8.9 & Toronto & 716240 & 40124 & $\mathrm{Dfb}$ & \\
\hline $\begin{array}{l}\text { Central African } \\
\text { Republic }\end{array}$ & 8.58 & 12.64 & 4.6 & 25.8 & Bangui & 646500 & 388 & Aw & \\
\hline Chad & 8.58 & 9.6 & 7.56 & 28.7 & N'Djamena & 647000 & 678 & $\mathrm{BSh}$ & \\
\hline Chile & 11.04 & 18.24 & 3.94 & 14.8 & Santiago & 855740 & 10248 & $\mathrm{Csb}$ & \\
\hline China & 11.02 & 9.72 & 12.38 & 17.5 & Shanghai & 583670 & 3947 & $\mathrm{Cfa}$ & \\
\hline
\end{tabular}




\begin{tabular}{|c|c|c|c|c|c|c|c|c|c|}
\hline Colombia & 7.4 & 11.8 & 3.06 & 13.3 & Bogota & 802220 & 4999 & $\mathrm{Cfb}$ & \\
\hline Comoros & 6.36 & 9.22 & 3.42 & 27.2 & Moroni & 670010 & 1171 & $\mathrm{Af}$ & Yes \\
\hline Congo & 7.7 & 10.74 & 4.62 & 25.5 & Brazzaville & 644500 & 2064 & Aw & \\
\hline Costa Rica & 7.92 & 13.54 & 2.28 & 22.7 & San Jose & 787620 & 7117 & $\mathrm{Am}$ & \\
\hline Cote d'Ivoire & 12.46 & 17.88 & 6.86 & 27.4 & Abidjan & 655780 & none & $\mathrm{Am}$ & \\
\hline Croatia & 18.88 & 28.98 & 9.44 & 13.7 & Zagreb & 142360 & 11434 & $\mathrm{Cfb}$ & \\
\hline Cuba & 14.48 & 22.14 & 6.8 & 24.3 & Havana & 782240 & 5170 & $\mathrm{Aw}$ & Yes \\
\hline Cyprus & 4.14 & 6.14 & 2.12 & 19.7 & Athalassa & 176070 & 25553 & $\mathrm{BSh}$ & Yes \\
\hline Czechia & 15.14 & 25.02 & 5.66 & 9.0 & Praha & 115180 & 15797 & $\mathrm{Cfb}$ & \\
\hline $\begin{array}{l}\text { Democratic People's } \\
\text { Republic of Korea }\end{array}$ & 11.08 & 13.66 & 8.66 & 11.0 & Pyongyang & 470580 & none & Dwa & \\
\hline $\begin{array}{l}\text { Democratic Republic of } \\
\text { the Congo }\end{array}$ & 5.9 & 8.64 & 3.18 & 25.8 & Kinshasa & 642100 & 326 & $\mathrm{Aw}$ & \\
\hline Denmark & 13.92 & 18.7 & 9.22 & 9.1 & Copenhagen & 61800 & 51380 & $\mathrm{Cfb}$ & \\
\hline Djibouti & 6 & 7.84 & 4.1 & 29.9 & Djibouti City & 631250 & 2568 & $\mathrm{BWh}$ & \\
\hline Dominican Republic & 8.98 & 14.88 & 3.12 & 26.4 & Santo Domingo & 784860 & 6203 & $\mathrm{Aw}$ & Yes \\
\hline Ecuador & 8.04 & 11.6 & 4.48 & 25.0 & Guayaquil & 842030 & 4067 & $\mathrm{Aw}$ & \\
\hline Egypt & 3.62 & 5.72 & 1.46 & 22.5 & Cairo & 623660 & 2196 & $\mathrm{BWh}$ & \\
\hline El Salvador & 12.36 & 20.7 & 4.86 & 25.3 & San Salvador & 786630 & 2859 & $\mathrm{Aw}$ & \\
\hline Equatorial Guinea & 15.76 & 23.4 & 6.88 & None & None & None & 12751 & $\mathrm{Af}$ & \\
\hline Eritrea & 9.4 & 14.1 & 4.72 & 19.6 & Asmara & 630210 & 452 & $\mathrm{BSh}$ & \\
\hline Estonia & 20.78 & 35.52 & 7.9 & 6.3 & Tallinn & 260380 & 13417 & $\mathrm{Dfb}$ & \\
\hline Eswatini & 12.38 & 18.68 & 6.48 & 22.1 & Manzini/Matsapa* & 683960 & 3189 & $\mathrm{Cwb}$ & \\
\hline Ethiopia & 7.52 & 11.6 & 3.44 & 16.7 & Addis Ababa & 634500 & 324 & $\mathrm{Cwb}$ & \\
\hline Fiji & 4.52 & 6.46 & 2.5 & 25.0 & Suva & 916900 & 3863 & $\mathrm{Af}$ & Yes \\
\hline Finland & 18.84 & 28.1 & 9.9 & 6.0 & Helsinki & 29740 & 41655 & $\mathrm{Dfb}$ & \\
\hline France & 19.86 & 27.1 & 12.88 & 12.5 & Paris & 71560 & 36258 & $\mathrm{Cfb}$ & \\
\hline Gabon & 6.82 & 10.3 & 3.24 & 26.5 & Libreville & 645000 & 7440 & $\mathrm{Am}$ & \\
\hline Gambia & 5.54 & 7.04 & 4.12 & None & None & None & 669 & $\mathrm{Aw}$ & \\
\hline Georgia & 6.84 & 11.68 & 2.4 & 11.0 & Tbilisi & 375490 & 2721 & $\mathrm{Cfa}$ & \\
\hline Germany & 13.88 & 20.54 & 7.48 & 9.8 & Berlin & 103840 & 37968 & $\mathrm{Cfb}$ & \\
\hline Ghana & 4.74 & 7.5 & 2.02 & 27.3 & Accra & 654720 & 1112 & Aw & \\
\hline Greece & 4 & 6.6 & 1.5 & 18.4 & Athens & 167160 & 21883 & $\mathrm{Csa}$ & \\
\hline Grenada & 1.66 & 2.38 & 0.94 & 27.4 & St. George's & 789580 & 7015 & Aw & Yes \\
\hline Guatemala & 3.02 & 4.36 & 1.72 & 19.3 & Guatemala City & 786410 & 2666 & $\mathrm{Cwb}$ & \\
\hline Guinea & 6.48 & 7.74 & 5.22 & 27.5 & Conakry & 618320 & 575 & $\mathrm{Am}$ & \\
\hline Guinea-Bissau & 4.3 & 4.98 & 3.66 & 27.2 & Bissau & 617660 & 509 & $\mathrm{Aw}$ & \\
\hline Guyana & 26.58 & 39.5 & 13.52 & 26.8 & Georgetown & 722547 & 2504 & Af & \\
\hline Haiti & 10.36 & 15.2 & 5.62 & 27.3 & Port-au-Prince & 784390 & 608 & Aw & Yes \\
\hline Honduras & 2.6 & 3.84 & 1.4 & 22.1 & Tegucigalpa & 787200 & 1681 & Cwa & \\
\hline Hungary & 24.92 & 39.6 & 11.64 & 11.6 & Budapest & 128430 & 11436 & $\mathrm{Cfb}$ & \\
\hline Iceland & 13.94 & 21.3 & 6.54 & 5.5 & Reykjavík & 40300 & 47910 & $\mathrm{Cfc}$ & Yes \\
\hline India & 16.86 & 18.04 & 15.58 & 24.9 & Mumbai & 430030 & 1048 & $\mathrm{Aw}$ & \\
\hline Indonesia & 3.62 & 5.06 & 2.2 & 25.8 & Jakarta & 967470 & 2250 & $\mathrm{Af}$ & Yes \\
\hline Iran & 7.22 & 8.76 & 5.6 & 18.4 & Tehran & 407540 & 4587 & $\mathrm{Csa}$ & \\
\hline Iraq & 2.82 & 3.34 & 2.24 & None & None & None & 4500 & $\mathrm{BWh}$ & \\
\hline Ireland & 11.86 & 18.9 & 4.88 & 9.8 & Dublin & 39690 & 49260 & $\mathrm{Cfb}$ & Yes \\
\hline Israel & 6.56 & 10.1 & 3.06 & 20.8 & Lod* & 401800 & 27484 & $\mathrm{Csa}$ & \\
\hline Italy & 7.7 & 11.46 & 4.16 & 15.9 & Roma & 162390 & 32040 & Csa & \\
\hline Jamaica & 2.1 & 3.3 & 0.9 & 28.0 & Kingston & 787930 & 4382 & $\mathrm{Aw}$ & Yes \\
\hline Japan & 22.38 & 32.22 & 12.96 & 16.6 & Tokyo & 476620 & 38732 & $\mathrm{Cfa}$ & Yes \\
\hline Jordan & 3.02 & 3.74 & 2.28 & 18.0 & Amman & 402700 & 3019 & $\mathrm{BSh}$ & \\
\hline Kazakhstan & 30.7 & 52.88 & 10 & 9.8 & Almaty & 368700 & 6991 & Dfa & \\
\hline Kenya & 3.54 & 5.66 & 1.42 & 19.3 & Nairobi & 637400 & 837 & $\mathrm{Cfb}$ & \\
\hline Kiribati & 14.2 & 23.32 & 5.38 & 27.3 & Tarawa Island & 914890 & 1325 & $\mathrm{Af}$ & Yes \\
\hline Kuwait & 2.16 & 2.56 & 1.66 & 26.9 & Kuwait City & 405820 & 35847 & $\mathrm{BWh}$ & \\
\hline Kyrgyzstan & 10.24 & 16.68 & 3.94 & 12.1 & Bishkek (Frunze) & 383530 & 782 & $\mathrm{Dfb}$ & \\
\hline $\begin{array}{c}\text { Lao People's } \\
\text { Democratic Republic }\end{array}$ & 9.1 & 12 & 6.22 & 25.2 & Viang Chan & 489400 & 1044 & Aw & \\
\hline Latvia & 25.38 & 44.78 & 8.92 & 7.5 & Riga & 264220 & 10585 & $\mathrm{Dfb}$ & \\
\hline Lebanon & 3.18 & 4.04 & 2.34 & 21.2 & Bayrouth & 401000 & 6176 & $\mathrm{Csa}$ & \\
\hline Lesotho & 19.78 & 16.44 & 22.88 & None & None & None & 918 & $\mathrm{Cwb}$ & \\
\hline Liberia & 6.46 & 6.86 & 6.06 & 27.4 & Harbel* & 656600 & 474 & $\mathrm{Am}$ & \\
\hline Libya & 5.14 & 7.74 & 2.4 & 21.0 & Tripoli & 620100 & 8052 & $\mathrm{BSh}$ & \\
\hline Lithuania & 39.74 & 70.14 & 13.52 & 7.0 & Vilnius & 267300 & 10677 & $\mathrm{Dfb}$ & \\
\hline Luxembourg & 14.44 & 20.66 & 8.26 & 9.9 & Luxembourg & 65900 & 91184 & $\mathrm{Cfb}$ & \\
\hline Madagascar & 4.28 & 6.08 & 2.48 & 18.7 & Antananarivo & 670830 & 406 & $\mathrm{Cwb}$ & Yes \\
\hline Malawi & 4.36 & 7 & 1.8 & 21.7 & Lilongwe & 675851 & 337 & Cwa & \\
\hline Malaysia & 5.34 & 7.58 & 3.02 & 27.8 & Kuala Lumpur & 486470 & 7568 & $\mathrm{Af}$ & \\
\hline
\end{tabular}


Arima \& Kikumoto (2020)

Global Suicide Rates and Climatic Temperature

\begin{tabular}{|c|c|c|c|c|c|c|c|c|c|}
\hline Maldives & 3.16 & 4.06 & 2.06 & 28.7 & Male & 435550 & 5842 & $\mathrm{Am}$ & Yes \\
\hline Mali & 4.96 & 7.16 & 2.8 & 27.7 & Bamako & 612910 & 603 & Aw & \\
\hline Malta & 7.12 & 11.28 & 2.94 & 18.9 & Luga/Qrendi* & 165970 & 18950 & $\mathrm{Csa}$ & Yes \\
\hline Mauritania & 3.9 & 5.84 & 1.92 & 25.5 & Nouakchott & 614420 & 995 & $\mathrm{BWh}$ & \\
\hline Mauritius & 8.86 & 14.02 & 3.76 & 24.1 & Plaisance & 619900 & 7065 & $\mathrm{Am}$ & Yes \\
\hline Mexico & 4.64 & 7.48 & 1.84 & 16.8 & Mexico City & 766793 & 8916 & $\mathrm{Cwb}$ & \\
\hline Micronesia & 11.26 & 15.48 & 6.92 & 27.9 & Pohnpei & 913480 & 2626 & $\mathrm{Af}$ & Yes \\
\hline Mongolia & 15.54 & 26.08 & 5.14 & -0.8 & Ulan Bator & 442920 & 2217 & $\mathrm{BSk}$ & \\
\hline Montenegro & 10.5 & 15.24 & 5.9 & 15.8 & Podgorica & 134620 & 5216 & $\mathrm{Cfa}$ & \\
\hline Morocco & 4.34 & 3.46 & 5.22 & 18.4 & Casablanca & 601550 & 2418 & $\mathrm{Csa}$ & \\
\hline Mozambique & 6.18 & 9.2 & 3.34 & 23.7 & Maputo & 673410 & 477 & Aw & \\
\hline Myanmar & 7.54 & 5.38 & 9.56 & 27.6 & Yangon & 480970 & 664 & $\mathrm{Am}$ & \\
\hline Namibia & 9.88 & 16.04 & 4.04 & 20.4 & Windhoek & 681100 & 4138 & $\mathrm{BSh}$ & \\
\hline Nepal & 11.26 & 12.04 & 10.48 & 19.0 & Kathmandu & 444540 & 487 & Cwa & \\
\hline Netherlands & 10.96 & 14.56 & 7.38 & 10.9 & Amsterdam & 62400 & 44610 & $\mathrm{Cfb}$ & \\
\hline New Zealand & 12.68 & 19.06 & 6.56 & 15.3 & Auckland & 931190 & 30371 & $\mathrm{Cfb}$ & Yes \\
\hline Nicaragua & 13.7 & 21.18 & 6.42 & 27.6 & Managua & 787410 & 1448 & $\mathrm{Aw}$ & \\
\hline Niger & 4.74 & 5.94 & 3.48 & 29.8 & Niamey & 610520 & 301 & $\mathrm{BSh}$ & \\
\hline Nigeria & 9.78 & 9.42 & 10.14 & 27.5 & Lagos & 652010 & 1843 & $\mathrm{Aw}$ & \\
\hline Norway & 12.64 & 16.8 & 8.42 & 7.1 & Oslo & 14920 & 74417 & $\mathrm{Dfb}$ & \\
\hline Oman & 3.88 & 5.78 & 0.9 & 28.2 & Muscat & 412560 & 15325 & $\mathrm{BWh}$ & \\
\hline Pakistan & 3.16 & 2.84 & 3.52 & 27.0 & Karachi & 417800 & 916 & $\mathrm{BWh}$ & \\
\hline Panama & 5.12 & 8.72 & 1.48 & 27.3 & Tocumen* & 787920 & 7829 & $\mathrm{Am}$ & \\
\hline Papua New Guinea & 6.06 & 8.52 & 3.52 & 26.9 & Port Moresby & 920350 & 1617 & Af & Yes \\
\hline Paraguay & 9.78 & 12.96 & 6.5 & 23.3 & Asuncion & 862180 & 3526 & $\mathrm{Cfa}$ & \\
\hline Peru & 4.82 & 7 & 2.68 & 19.1 & Lima & 846280 & 4220 & $\mathrm{BWh}$ & \\
\hline Philippines & 2.96 & 4.14 & 1.8 & 27.2 & Maynila* & 984300 & 1853 & $\mathrm{Af}$ & Yes \\
\hline Poland & 17.04 & 30.16 & 4.78 & 9.0 & Warszawa & 123750 & 10215 & $\mathrm{Cfb}$ & \\
\hline Portugal & 11.98 & 18.98 & 5.54 & 16.8 & Lisboa & 85350 & 19316 & $\mathrm{Csa}$ & \\
\hline Qatar & 5.16 & 6.72 & 0.92 & 28.2 & Doha & 411700 & 59123 & $\mathrm{BWh}$ & \\
\hline Republic of Korea & 26.18 & 35.88 & 16.42 & 12.9 & Seoul & 471080 & 20374 & Dwa & \\
\hline Republic of Moldova & 17.76 & 31.32 & 5.22 & 10.8 & Kishinev & 338150 & 1487 & $\mathrm{Dfb}$ & \\
\hline Romania & 12.34 & 21.02 & 4.16 & 11.9 & Bucharest & 154200 & 6675 & $\mathrm{Cfa}$ & \\
\hline Russian Federation & 41.46 & 74.28 & 12.86 & 6.3 & Moscow & 276120 & 8441 & $\mathrm{Dfb}$ & \\
\hline Rwanda & 9.5 & 12.62 & 6.5 & None & None & None & 472 & $\mathrm{Aw}$ & \\
\hline Saint Lucia & 6.22 & 10.98 & 1.62 & 27.6 & Vieux Fort* & 789480 & 7221 & $\mathrm{Af}$ & Yes \\
\hline $\begin{array}{l}\text { Saint Vincent and the } \\
\text { Grenadines }\end{array}$ & 4.92 & 8.5 & 1.26 & None & None & None & 5711 & $\mathrm{Af}$ & Yes \\
\hline Samoa & 5.74 & 8.78 & 2.44 & 26.7 & Apia & 917590 & 3044 & $\mathrm{Af}$ & Yes \\
\hline Sao Tome and Principe & 2.08 & 2.76 & 1.4 & 26.8 & Sao Tome & 619310 & none & As & Yes \\
\hline Saudi Arabia & 3.12 & 4.5 & 1.34 & 27.3 & Riyadh & 404380 & 16900 & $\mathrm{BWh}$ & \\
\hline Senegal & 6.98 & 10.54 & 3.58 & 24.6 & Dakar & 616410 & 1110 & $\mathrm{BSh}$ & \\
\hline Serbia & 17.26 & 25.26 & 9.62 & 13.1 & Beograd & 132740 & 4782 & $\mathrm{Cfa}$ & \\
\hline Seychelles & 9.18 & 15.92 & 2.48 & 27.3 & Anse des Genets* & 639800 & 11333 & $\mathrm{Af}$ & Yes \\
\hline Sierra Leone & 9.94 & 11.76 & 8.2 & 27.3 & Freetown & 618560 & 404 & $\mathrm{Am}$ & \\
\hline Singapore & 10.58 & 14.16 & 7.1 & 27.8 & Singapore & 486980 & 40278 & $\mathrm{Af}$ & Yes \\
\hline Slovakia & 13.54 & 23.64 & 3.98 & 11.2 & Bratislava & 118160 & 13849 & $\mathrm{Cfb}$ & \\
\hline Slovenia & 23.96 & 37.72 & 10.6 & 11.2 & Luubluana & 140150 & 19985 & $\mathrm{Cfb}$ & \\
\hline Solomon Islands & 6.84 & 9.32 & 4.24 & 27.0 & Honiara & 915201 & 1317 & $\mathrm{Af}$ & Yes \\
\hline Somalia & 4.64 & 6.2 & 3.1 & 31.3 & Berbera* & 631600 & 296 & $\mathrm{BWh}$ & \\
\hline South Africa & 11.8 & 18.56 & 5.24 & 16.0 & Johannesburg & 683680 & 5447 & $\mathrm{Cwb}$ & \\
\hline South Sudan & 4.18 & 5.48 & 2.86 & 25.3 & Juba & 629410 & 1433 & Aw & \\
\hline Spain & 8.1 & 12.26 & 4.1 & 14.5 & Madrid & 82210 & 26434 & $\mathrm{Csa}$ & \\
\hline Sri Lanka & 20.08 & 31.88 & 8.74 & 27.3 & Colombo & 434660 & 2210 & $\mathrm{Af}$ & Yes \\
\hline Sudan & 8.08 & 12.18 & 3.98 & 30.5 & Khartoum & 627210 & 1266 & $\mathrm{BWh}$ & \\
\hline Suriname & 24.72 & 38.08 & 11.26 & 26.3 & Paramaribo & 8120016 & 5834 & $\mathrm{Af}$ & \\
\hline Sweden & 15.56 & 20.62 & 10.5 & 7.8 & Stockholm & 24640 & 47460 & $\mathrm{Dfb}$ & \\
\hline Switzerland & 18.62 & 24.36 & 13.02 & 9.9 & Zurich & 66600 & 65779 & $\mathrm{Cfb}$ & \\
\hline Syrian Arab Republic & 1.72 & 2.68 & 0.76 & 18.0 & Aleppo & 400070 & 1464 & $\mathrm{Csa}$ & \\
\hline Tajikistan & 2.68 & 3.9 & 1.42 & 15.9 & Dusanbe & 388360 & 598 & Dsb & \\
\hline Thailand & 14.44 & 22.64 & 6.56 & 29.1 & Bangkok & 484550 & 4130 & Aw & \\
\hline $\begin{array}{c}\text { Republic of North } \\
\text { Macedonia }\end{array}$ & 7.3 & 10.62 & 3.96 & 12.4 & Skopje & 135860 & 3853 & $\mathrm{Cfb}$ & \\
\hline Timor-Leste & 6 & 8.14 & 3.8 & 27.4 & Dili & 973900 & 2663 & $\mathrm{Aw}$ & Yes \\
\hline Togo & 9.6 & 12.86 & 6.38 & 27.3 & Lome & 653870 & 482 & Aw & \\
\hline Tonga & 3.52 & 4.14 & 2.88 & 24.2 & Tongatapu & 917920 & 3193 & $\mathrm{Af}$ & Yes \\
\hline Trinidad and Tobago & 13.78 & 22.44 & 5.3 & 26.9 & Piarco* & 789700 & 14587 & $\mathrm{Am}$ & Yes \\
\hline Tunisia & 3.5 & 4.78 & 2.24 & 19.5 & Tunis & 607150 & 3535 & $\mathrm{Csa}$ & \\
\hline Turkey & 7.56 & 11.92 & 3.32 & 11.3 & Ankara & 171300 & 8646 & $\mathrm{Csb}$ & \\
\hline
\end{tabular}


Arima \& Kikumoto (2020)

Global Suicide Rates and Climatic Temperature

\begin{tabular}{|c|c|c|c|c|c|c|c|c|c|}
\hline Turkmenistan & 8.46 & 13.2 & 3.86 & 17.6 & Ashgabat & 388800 & 3786 & $\mathrm{BSk}$ & \\
\hline Uganda & 10.38 & 10.7 & 10.1 & 21.9 & Kampala & 636800 & 474 & $\mathrm{Af}$ & \\
\hline Ukraine & 28.8 & 51.82 & 8.9 & 9.0 & Kiev & 333450 & 2363 & $\mathrm{Dfb}$ & \\
\hline United Arab Emirates & 2.98 & 3.86 & 0.84 & 28.4 & Dubai & 411940 & 37801 & $\mathrm{BWh}$ & \\
\hline $\begin{array}{l}\text { United Kingdom of } \\
\text { Great Britain and } \\
\text { Northern Ireland }\end{array}$ & 8.54 & 12.84 & 4.42 & 12.4 & London & 37790 & 40276 & $\mathrm{Cfb}$ & Yes \\
\hline $\begin{array}{c}\text { United Republic of } \\
\text { Tanzania }\end{array}$ & 5.7 & 8.14 & 3.3 & 26.1 & Dar es Salaam & 638940 & 667 & Aw & \\
\hline $\begin{array}{l}\text { United States of } \\
\text { America }\end{array}$ & 13.34 & 20.76 & 6.1 & 13.2 & New York City & 725030 & 47022 & $\mathrm{Cfa}$ & \\
\hline Uruguay & 17.42 & 28.1 & 7.42 & 16.4 & Paso de Carrasco* & 865800 & 9876 & $\mathrm{Cfa}$ & \\
\hline Uzbekistan & 6.84 & 9.68 & 4 & 16.1 & Tashkent & 384570 & 1308 & $\mathrm{Csa}$ & \\
\hline Vanuatu & 5.42 & 7.88 & 2.88 & 24.7 & Port Vila & 915570 & 2383 & Af & Yes \\
\hline Venezuela & 5.14 & 8.68 & 1.62 & 27.7 & Caracas & 804150 & 8760 & Aw & \\
\hline Viet Nam & 6.98 & 10.46 & 3.62 & 27.4 & Ho Chi Minh & 489000 & 1163 & Aw & \\
\hline Yemen & 8.16 & 10.94 & 5.26 & 19.7 & Sanaa & 414040 & 1078 & BWk & \\
\hline Zambia & 6.62 & 9.52 & 3.8 & 20.8 & Lusaka & 676650 & 1100 & Cwa & \\
\hline Zimbabwe & 12 & 18.32 & 6 & 19.1 & Harare & 677750 & 837 & $\mathrm{Cwb}$ & \\
\hline
\end{tabular}

* in "the city at which the weather data we used" means that the city is not the most populous one. 


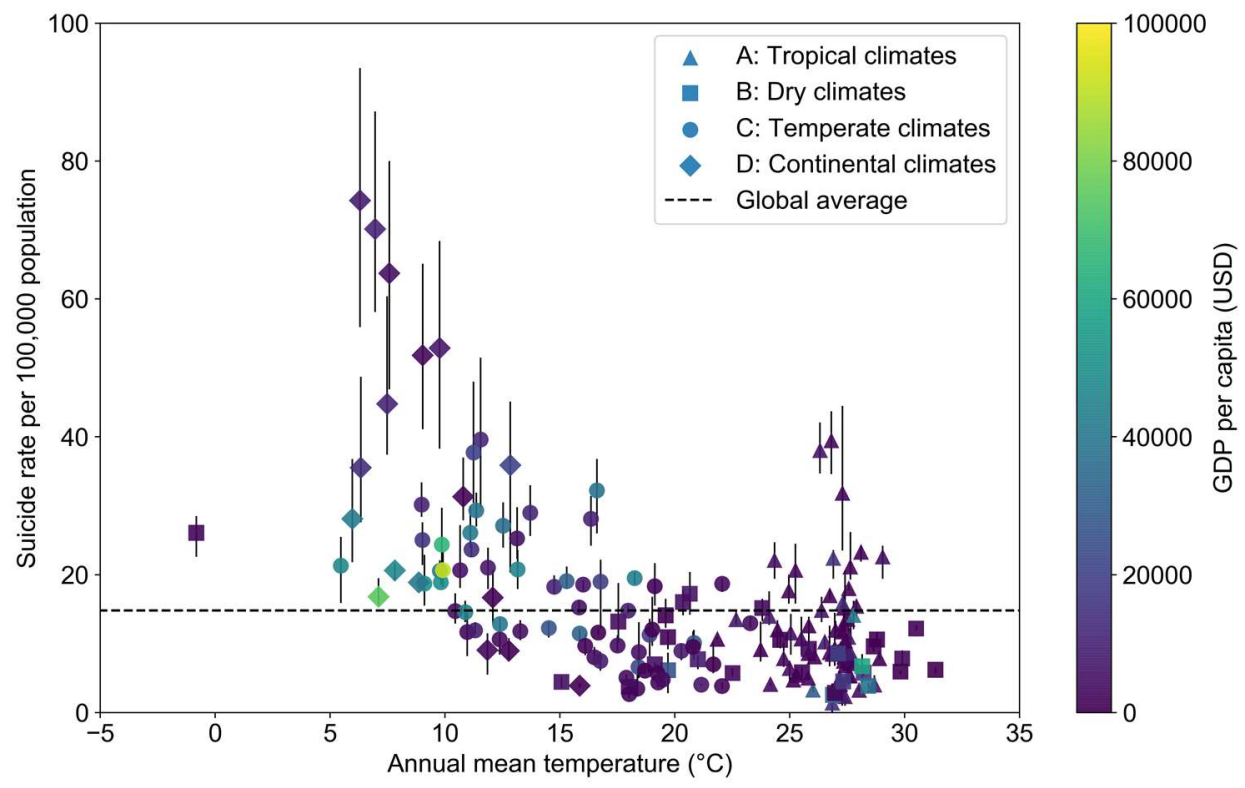

a. Male

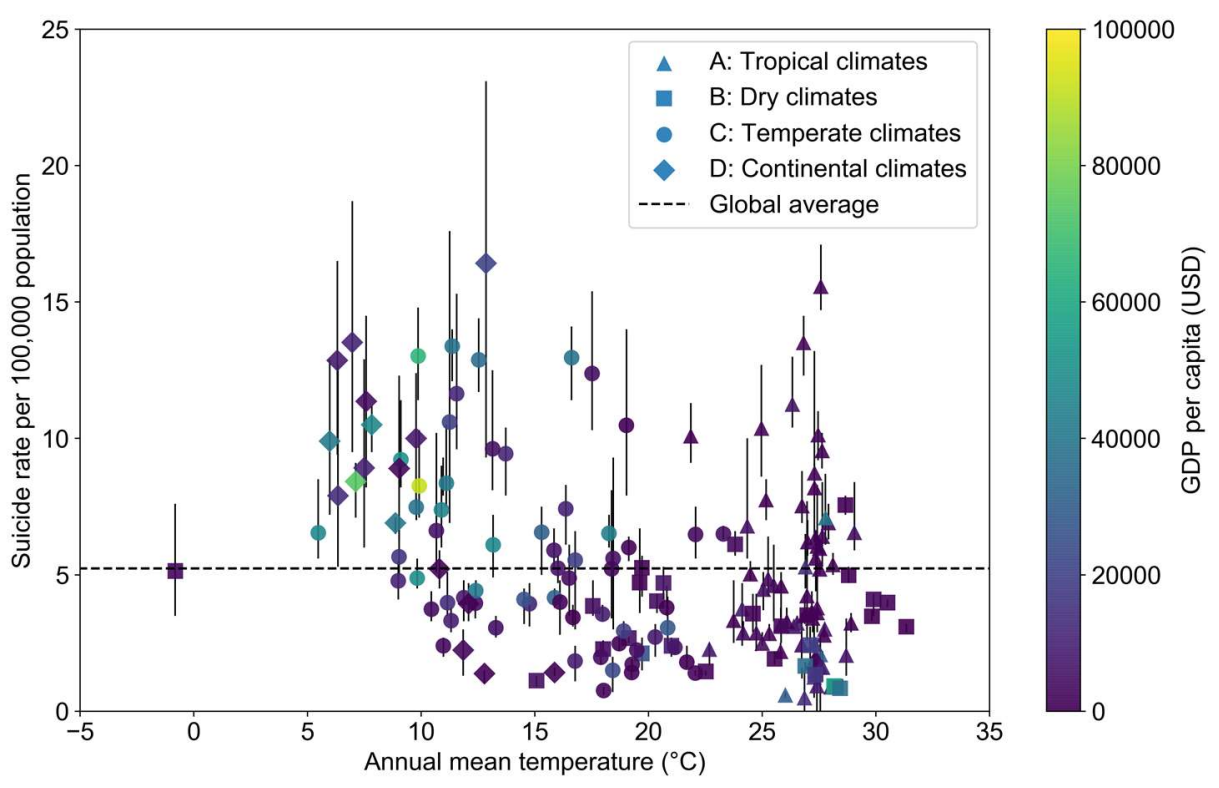

b. Female

Extended Data Fig. 1| Correlation between annual mean temperature and global suicide rate for males and females. Countries have been categorized according to the Köppen-Geiger classification, denoted by markers colored according to the average GDP per capita in the years considered. Error bars indicate the minimum-maximum range in annual suicide rate for each country. The broken line shows global average suicide rate (14.8 and 5.23 per 100,000 males and females, respectively). 


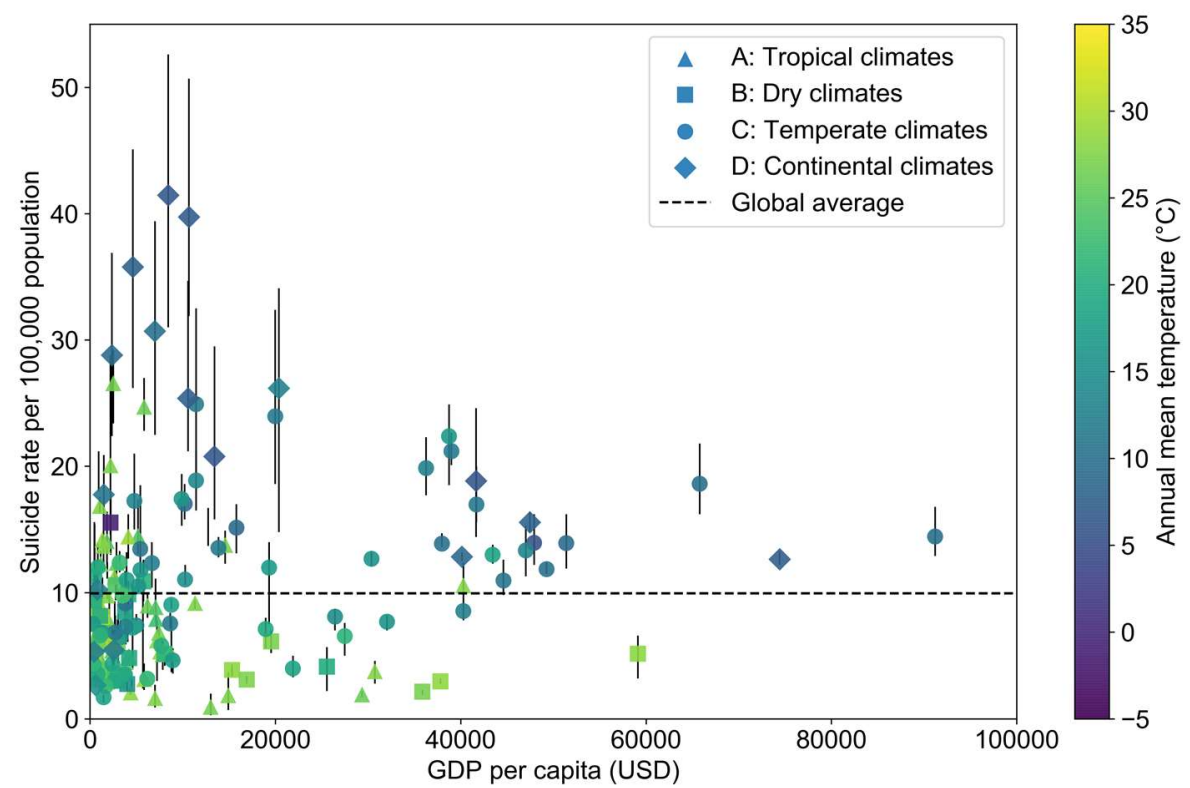

Extended Data Fig. 2| Correlation between average GDP per capita and global suicide rate for both sexes. Countries have been categorized according to the Köppen-Geiger classification, denoted by markers colored according to average GDP per capita in the years considered. Error bars indicate the minimum-maximum range in annual suicide rate for each country. The broken line shows average global suicide rate ( 9.94 per 100,000 people).

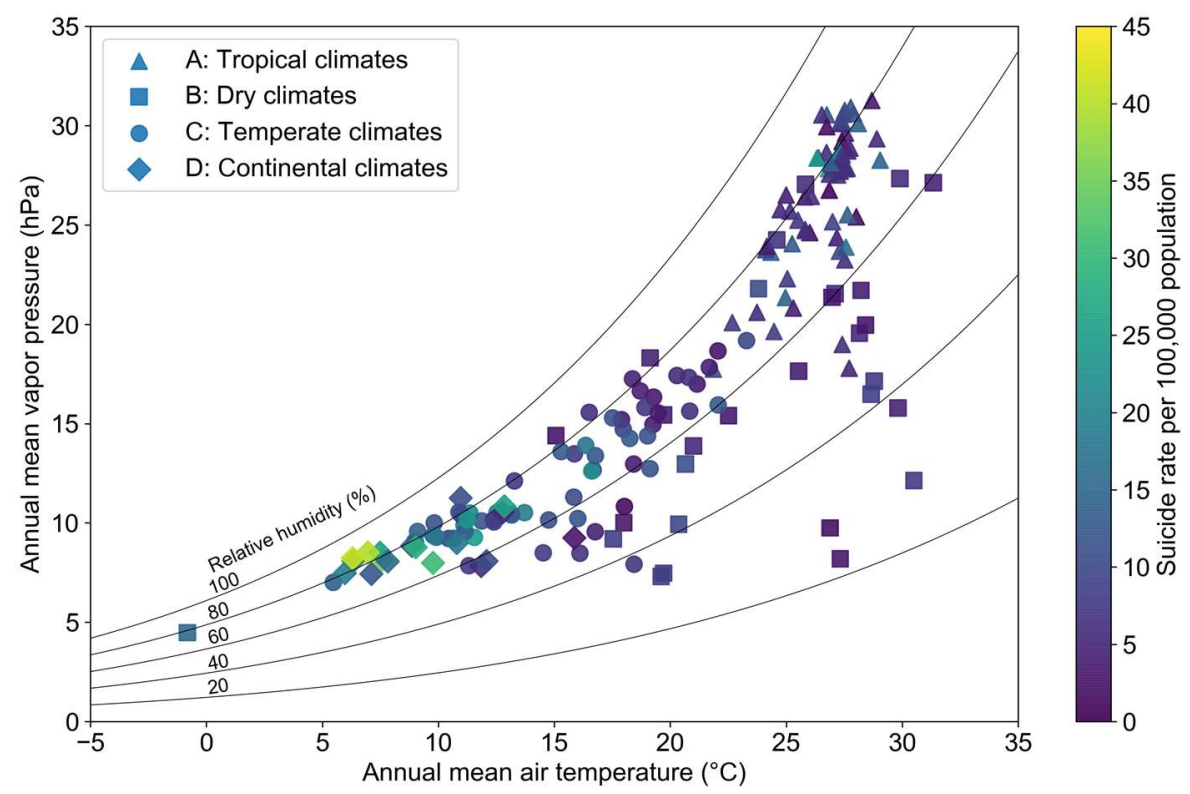

Extended Data Fig. 3| Correlation between annual mean temperature and vapor pressure worldwide. Countries have been categorized according to the Köppen-Geiger classification, denoted by markers colored according to the suicide rate for both sexes. 


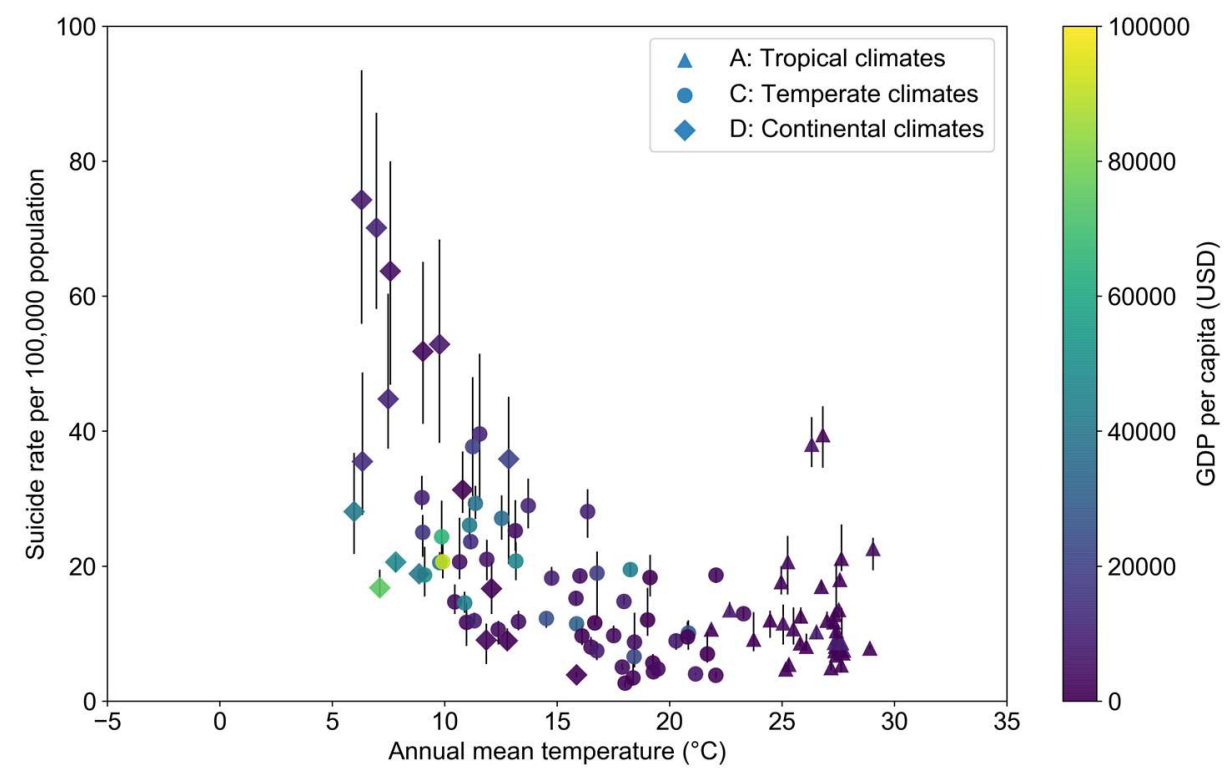

a. Male

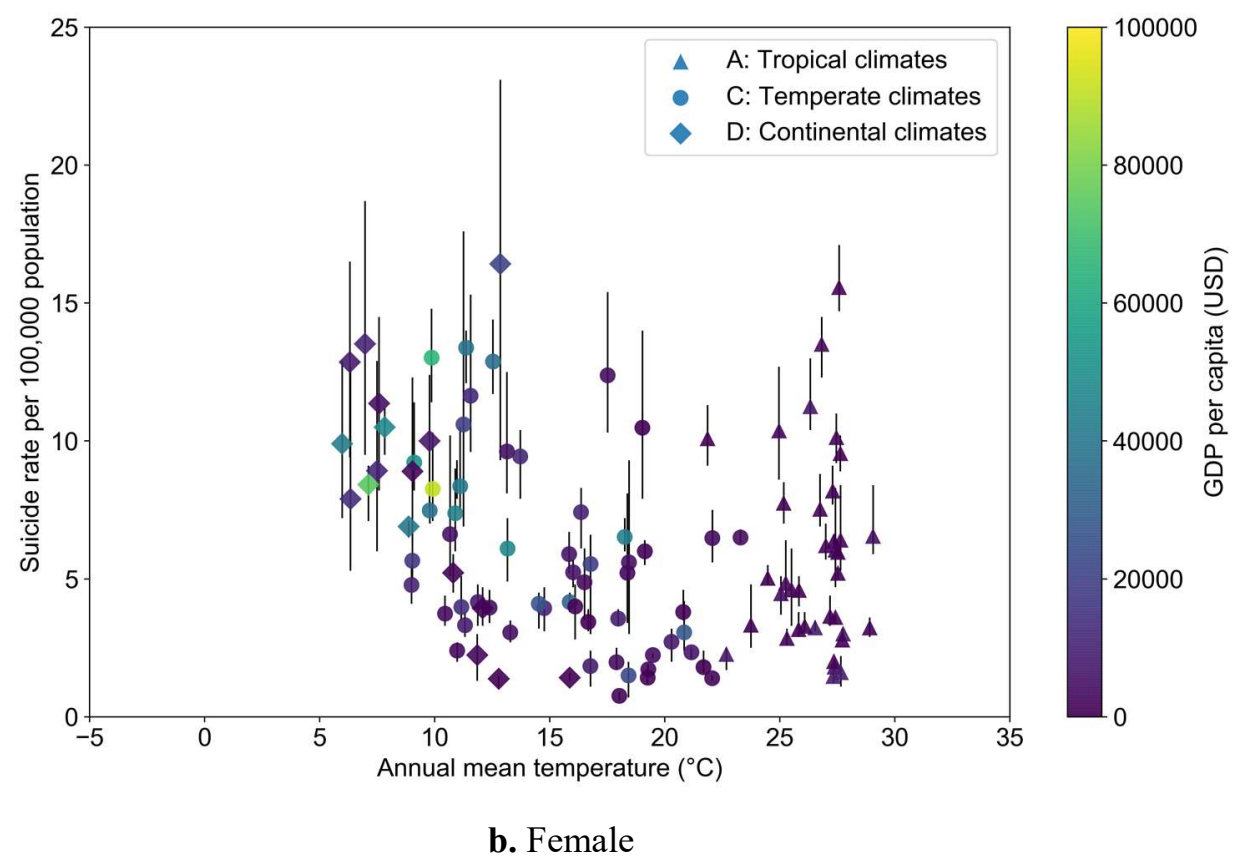

Extended Data Fig. $4 \mid$ Correlation between annual mean temperature and suicide rate for males and females globally, excluding countries in dry zones and island countries. Countries have been categorized according to the Köppen-Geiger classification, denoted by markers colored according to average GDP per capita in the years considered. Error bars indicate minimum-maximum range in annual suicide rate for each country. 